\title{
Draping of strongly flow-aligned interplanetary magnetic field about the magnetopause
}

\author{
S.M.Petrinec \\ Lockheed Martin Advanced Technology Center, Palo Alto, CA, 94304 USA
}

\begin{abstract}
Many dynamic processes of the magnetosphere are directly driven by the solar wind and the occurrence of magnetic merging at the magnetopause. The location of magnetopause magnetic merging, or reconnection, is now fairly well understood when the interplanetary magnetic field (IMF) contains large $B_{y}$ and $B_{z}$ components in relation to the $B_{x}$ component (in Geocentric Solar Magnetospheric (GSM) coordinates). However, when the IMF contains a large $X$-component (i.e., is closely flow-aligned), it is not yet well understood how the shocked IMF drapes about the magnetopause, and how this affects the occurrence and location of magnetic merging. In this initial study, we examine from observations how a nearly flow-aligned IMF drapes about the magnetopause. The results of this study are expected to be useful for comparisons with both analytic and global numerical models of the magnetosheath magnetic field.
\end{abstract}

Keywords: Magnetosheath, magnetopause, IMF draping

Corresponding author: Steven Petrinec

steven.m.petrinec@Imco.com

$+1-650-354-5562$ 


\section{Introduction.}

The location of magnetic reconnection at the dayside magnetopause is now fairly well understood when the interplanetary magnetic field (IMF) contains large $B_{y}$ and $B_{z}$ GSM-components in relation to the $B_{x}$ component (e.g., Trattner et al., [2007, 2012]; Trenchi et al., [2008]; Fuselier et al., [2011]). However, it is not well understood where reconnection occurs when a relatively large IMF $B_{x}$ component exists (also described in the literature as a nearly radial IMF, as it is closely oriented along a direction that intersects the center of the Sun). This is because there is poor understanding of how the magnetic field drapes about the magnetosphere for an IMF with a dominant $x$-component. Therefore, in order to improve understanding of where magnetic reconnection at the magnetopause is likely to occur, it is important to understand IMF draping under all solar wind conditions. For example, for strongly solar wind flow-aligned IMF configurations, it is easy to envision that a draped IMF may be oriented in one direction over part of the magnetopause (e.g., southward, causing dayside magnetopause reconnection), while simultaneously oriented the opposite direction over other regions of the magnetopause (e.g., northward, leading to reconnection tailward of the cusp). This type of behavior has recently been noted in a numerical model by Tang et al., [2013] for an exactly flow-aligned IMF. The separation between, for example, northward and southward draped magnetosheath magnetic field in general is not yet well understood in the case of strongly radial IMF.

However, there are many challenges involved when studying strongly flow-aligned IMF conditions. The first involves the probability of finding such solar wind intervals. Using the solar wind flow velocity $(\boldsymbol{V})$ to define a 'polar' direction, the angle between the IMF $(\boldsymbol{B})$ and velocity is defined as $\cos ^{-1}\left(\boldsymbol{V} \cdot \boldsymbol{B} /\left(V B_{T}\right)\right)$ (also often described as a cone angle). The fraction of the unit sphere covered by cone angles close to the 'pole' is small, and so the number of intervals when the IMF lies close to flow velocity vector is also small (described more quantitatively below). For completeness, the 'azimuthal' angle (also called the IMF clock angle) is often described as $\tan ^{-1}\left(B_{z} / B_{y}\right)$. 
The second challenge involves the steadiness of the IMF, and hence the cone angle. When the cone angle is closer to $90^{\circ}$, small random variations in the IMF do not change these angles significantly. However, when the angle is close to $0^{\circ}$ (or $180^{\circ}$ ), small random variations can significantly change both the cone and clock angles.

The third challenge is how to propagate the solar wind from its point of measurement (often close to the $L_{1}$ Lagrangian point) to the bow shock, and then through the magnetosheath. The simplest method is to calculate the propagation time using the distance of the solar wind monitor from a model bow shock and divide by the solar wind velocity; and then add another small time increment using the distance from the model bow shock to the spacecraft near the magnetopause, divided by a slowed solar wind speed (e.g., V/4). More advanced (and presumably more accurate) models account for the phase front using the IMF (Weimer et al., [2002, 2003]; Bargatze et al., [2005]; Haaland et al., [2006]; Weimer and King [2008]). However, when the IMF is strongly flow-aligned, it can be very challenging to determine the most appropriate propagation time using these more involved models.

Finally, the IMF intersection with the normal to the bow shock can provide an additional challenge. The region immediately downstream of the quasi-parallel shock is known to be quite turbulent (e.g., Greenstadt and Fredricks [1979]; Kan and Swift [1983]; Scholer et al., [1993]), due to shock reformation, convection of foreshock instabilities into the magnetosheath, and the in situ excitation of various wave modes. For nearly radial IMF, the quasi-parallel bow shock covers most of the dayside region, and strongly influences the magnetosheath flow downstream. However, it is also known that despite the turbulent magnetosheath field on the downstream side of the bow shock, the shocked IMF is aligned tangential to the magnetopause as the plasma flow approaches this surface. It is not clear whether the components tangential to the magnetopause are as turbulent as they are further upstream from the magnetopause, or if they retain some of the large-scale directionality of the IMF configuration. 
There have been some observational studies which have examined the effects of nearly flow-aligned IMF on the magnetosphere. Merka et al. [2003] noted that under such conditions the magnetopause was located significantly further from the Earth than predicted by statistical models. However, the magnetosheath has also been found to be thinner under conditions of strongly flow-aligned IMF as compared to other IMF orientations (e.g., Jelínek et al. [2010]; Suvorova et al. [2010]). Shue et al. [2009] investigated a case with multiple THEMIS spacecraft wherein the magnetopause was found to rebound, with fast sunward flows confined to a relatively small region of the magnetopause. Farrugia et al. [2010] examined an interval when Wind was in the magnetosheath and ACE in the solar wind. The interplanetary flow was within $15^{\circ}$ of the flow vector, and fair agreement was found with an analytic formulation of magnetic field components derived from perturbations to the Spreiter and Rizzi model (Spreiter and Rizzi [1974]). In addition, pulsed high-speed flows exceeding that of the solar wind were observed in the dawnside boundary layer, which were discussed in terms of the Kelvin-Helmholtz instability.

For this study, magnetosheath magnetic fields draping the magnetopause are examined when the IMF cone angle $\left(\cos ^{-1}\left(B_{x-a G S E} / B_{T}\right)\right)$ lies between $5^{\circ}$ and $15^{\circ}$ or between $165^{\circ}$ and $175^{\circ}$ from the nominal aberrated solar wind direction (aberrated Geocentric Solar Ecliptic (GSE) coordinates). These ranges of IMF configurations are chosen so that a sufficient number of relatively strongly flow-aligned cases can be investigated, while the small asymmetry of the IMF from the flow direction is used to examine how the draped IMF differs on the two sides of the magnetosphere.

As mentioned above, the restrictions on the IMF cone angle exclude a large percentage of the possible IMF configurations. If the distribution of IMF orientations were completely isotropic, then one would expect that $3.03 \%$ of the intervals would lie within the cone angle ranges. However, the actual solar wind IMF configuration at $1 \mathrm{AU}$ lies preferentially along a Parker-spiral angle of $45^{\circ}$, and favors the ecliptic plane. The influence of the Parker-spiral angle is shown using the 1-minute resolution OMNI IMF 
data set for the interval April 1996 - October 2005 in Figure 1a., where peaks are observed at $\sim 45^{\circ}$ and $\sim 135^{\circ}$. Folding the cone angles such that $\theta=\cos ^{-1}\left(\left|B_{x-a G S E}\right| / B_{T}\right)$, the percentage of time that the IMF vector is between $5^{\circ}$ and $15^{\circ}$ from the flow direction is $3.38 \%$ (Figure $1 b$ ).
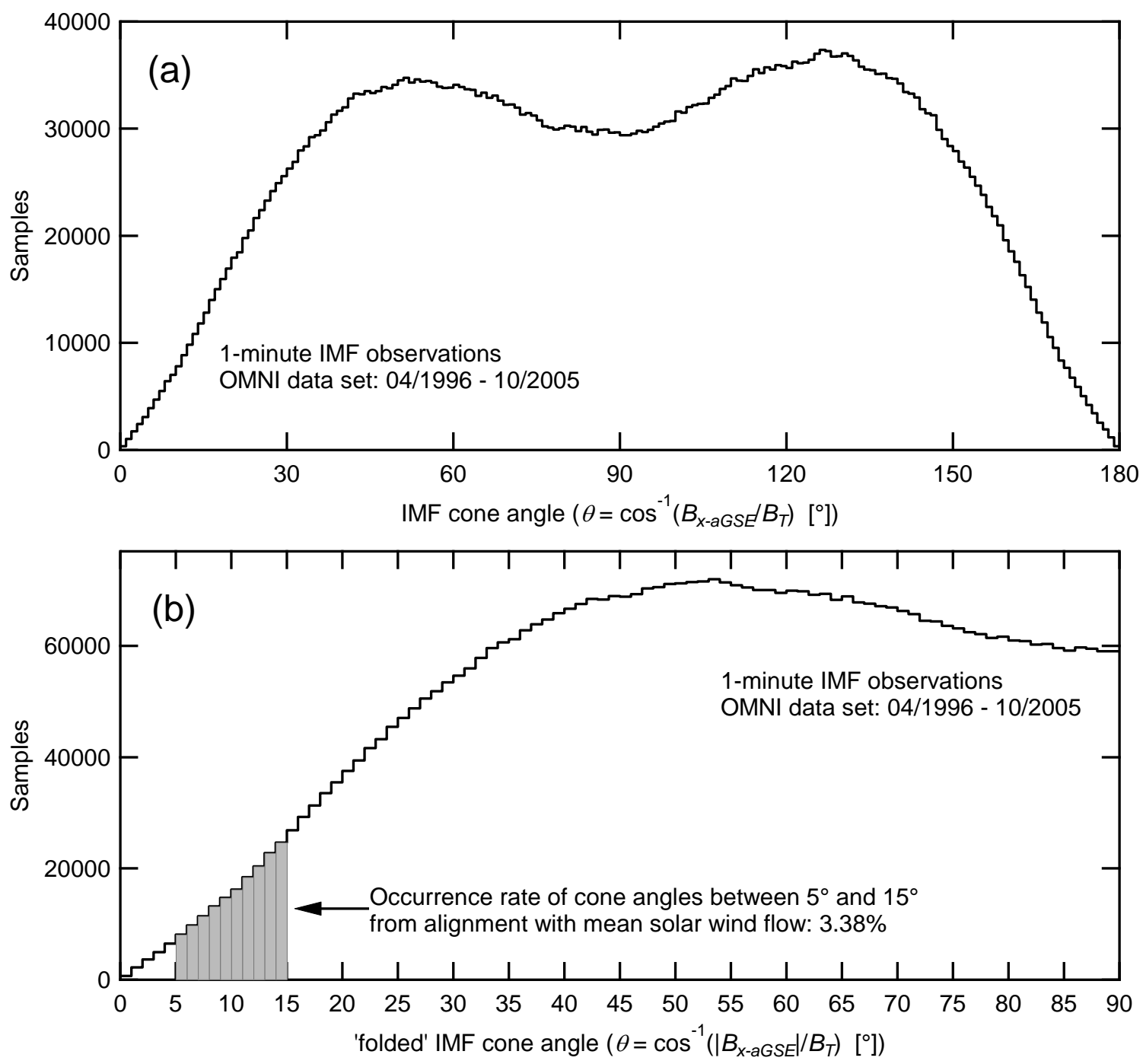

Fig.1. a) Histogram of the occurrence of IMF cone angles $\left(\theta=\cos ^{-1}\left(B_{x-a_{-} G S E} / B_{T}\right)\right)$ over a $9 \frac{1}{2}$ year span. $b)$ Histogram of 'folded' IMF cone angles $\left(\theta=\cos ^{-1}\left(\left|B_{x-a_{-} G S E}\right| / B_{T}\right)\right)$, including the summed occurrence of 'folded' cone angles between $5^{\circ}$ and $15^{\circ}$.

It is noted that periods of nearly flow-aligned IMF of long-duration (a few to several hours) have been catalogued in various investigations (Neugebauer and Goldstein [1997]; Neugebauer et al. [1997]; Watari et al., [2005]; Pi et al., [2014]), and the variation in occurrence frequency has been examined in 
terms of the solar cycle, with varying results. The focus of the present study, however, is not concerned with the distribution of IMF cone angles as a function of solar cycle phase.

Although there have been several studies of the consequences of perfectly flow-aligned IMF (e.g., Spreiter and Rizzi [1974] (and references therein); Tang et al., [2013]), there is relatively little knowledge as to how the IMF drapes about the magnetosphere when a relatively strong (but not perfectly flowaligned) IMF $B_{x-a G S E}$ component exists. As mentioned above, the work of Farrugia et al., [2010] provides an excellent case study for an IMF orientation of $\sim 15^{\circ}$ deviation from the solar wind flow direction; tracked using Wind observations in the magnetosheath and ACE solar wind observations. Farrugia et al. also used perturbation techniques to derive magnetic field components, which compared favorably with the magnetosheath observations. In general, it is reasonable to postulate that a controlling factor would be where the IMF intersects normal to the bow shock (i.e., where $\theta_{\mathrm{Bn}}=0^{\circ}$ ). If the IMF were exactly aligned with the solar wind flow, then the invocation of symmetry arguments (and neglecting the growth of instabilities) would lead one to conclude that the IMF would diverge about the magnetopause standoff location. Continuing this line of reasoning, the location of IMF intersection normal to the bow shock for a more general IMF configuration (but still strongly flow-aligned) is investigated here.

The location where the IMF is normal to the bow shock is calculated with a simple model of the dayside bow shock (Farris et al., [1991]), with the focus centered at the center of the Earth, and where the standoff distance $r_{0}=13.7 R_{E}$ and $\varepsilon=0.81$. A rigorous derivation of the bow shock locations at which the IMF is normal to the bow shock surface is provided in the Appendix. For $\psi=5^{\circ},\{x, y\}=\{13.6,-2.2\} R_{E}$, and for $\psi=15^{\circ},\{x, y\}=\{12.8,-6.6\} R_{E}$ (the negative root of Eq. A8 in the Appendix is chosen for $y$, to keep with the familiar picture of the Parker-spiral orientation).

With the results of this calculation, a couple of simple schematics representing potential IMF draping about the magnetosphere within the plane defined by the IMF and the $x_{a G S E}$ axis are shown in Figure 2. The direction towards the Sun is at the top of the Figure, the red curve represents the bow 
shock, and the blue curve represents the magnetopause. The upper panel depicts a simple divergence of the magnetic field at the magnetopause at the location along the direction where the IMF is normal to the bow shock ( $\psi=5^{\circ}$ shown in violet, $\psi=15^{\circ}$ in green). For visualization purposes, the drapings are offset slightly from the magnetopause and from each other. Also, in the schematic the IMF is depicted pointing anti-sunward, whereas it is understood that is equally probable that the IMF could be pointed Sunward. The lower panel of Figure 2 illustrates a scenario where the IMF divergence occurs at some point further downstream at the magnetopause. 

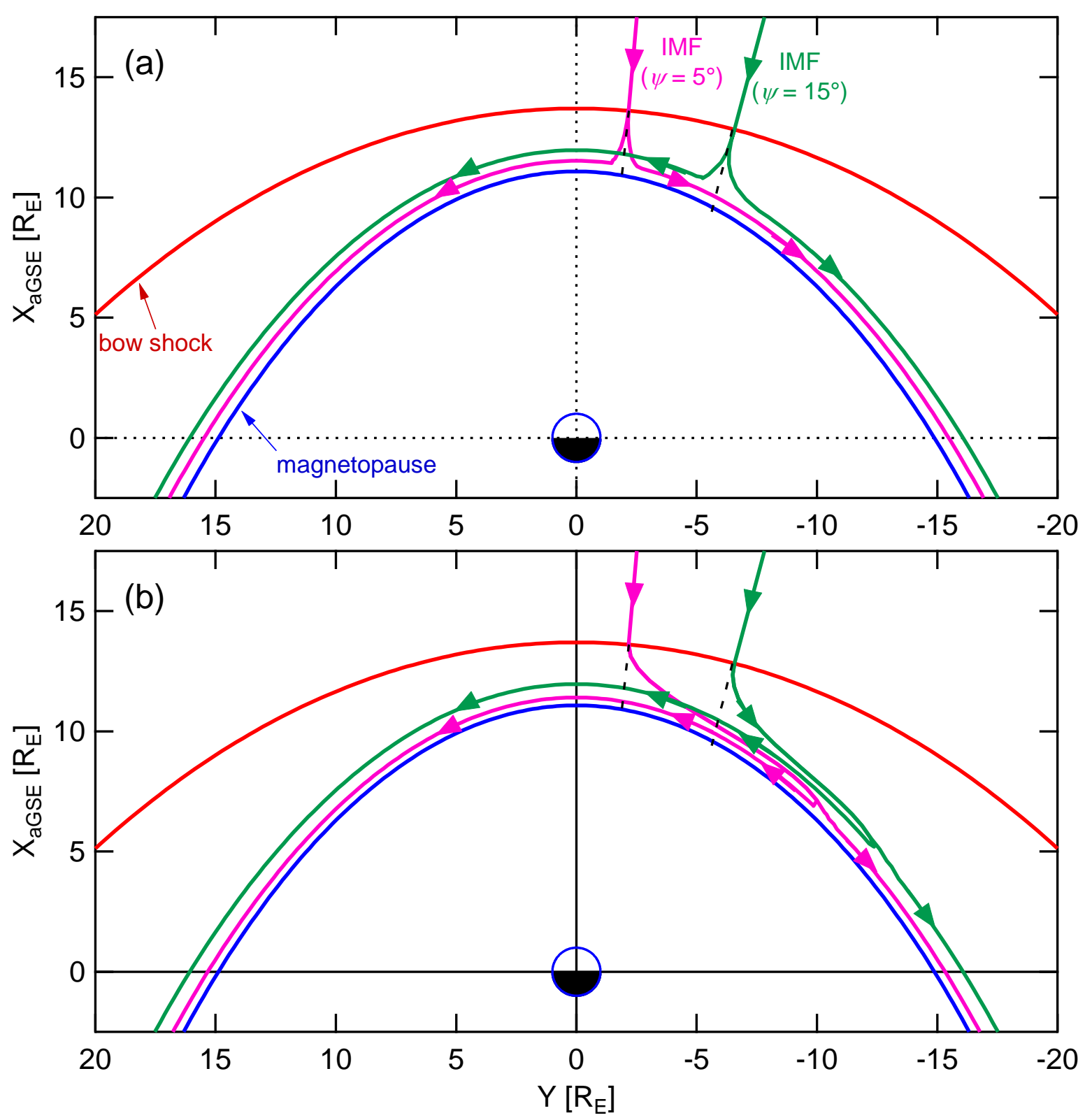

Fig.2. a) Schematic of field line draping, if the divergence of the field at the magnetopause occurs on average at the point where the IMF lies along the normal to the bow shock $\left(\theta_{\mathrm{Bn}}=0^{\circ}\right)$; projected along the same direction to the magnetopause. b) Schematic of field line draping along the magnetopause, if the magnetosheath flow causes divergence to occur further down along the flank. 


\section{Data sets}

A compiled list of 5757 magnetopause crossings from the Geotail spacecraft spanning the interval April 1996 - October 2005 are used in this study. These are matched with solar wind observations propagated from the Wind spacecraft to the Geotail location. The Geotail orbit is relatively close to the equatorial plane, and since the IMF tends to be close to the ecliptic plane, this helps to maximize the number of useful crossings for this study. Nevertheless, there are several criteria which greatly reduce the number of intervals to be examined. The first is the restriction of the IMF cone angle to the ranges $5^{\circ}$ $-15^{\circ}$ and $165^{\circ}-175^{\circ}$. The second criterion considers the distance of the spacecraft from the plane defined by the IMF and the $x_{a G S E}$ axis. For this study, only those intervals for which the spacecraft is within $\pm 3 R_{E}$ from this plane are considered. Also, only intervals on the dayside are considered. Lastly, there must be significant sampling of the magnetosheath magnetic field. Some intervals are too brief; i.e., the spacecraft does not exit the magnetosheath boundary layer for a sufficient amount of time (defined here to be at least 90 seconds) before crossing back into the magnetosphere. Geotail magnetic field and plasma spectrograms are examined by eye to determine the intervals when the spacecraft samples the magnetosheath proper just outside the magnetopause and magnetosheath boundary layer, and the mean magnetic field components are calculated for these intervals (an interval being defined as 3 minutes, but not less than 90 seconds). From the original list of 5757 Geotail magnetopause crossings, only 28 intervals meet all criteria. Of these, five intervals have $Y_{G e o t a i l}>+4 R_{E}$, and are far on the opposite flank from where we expect the magnetic field divergence to occur. Thus, we examine in the next section the remaining 23 Geotail magnetosheath intervals. 


\section{Analysis}

The general strategy of this analysis is to examine the magnetosheath magnetic field just upstream of the magnetopause when the propagated IMF is between $5^{\circ}$ and $15^{\circ}$ (or $165^{\circ}-175^{\circ}$ ) from the $x_{a G S E}$ axis. For each case, the coordinate system is rotated about the $x_{a G S E}$ axis such that the IMF lies within a plane such as depicted in Figure 2. It is expected that the draped IMF (restricted to those cases where observations are made close to this rotated $x y$-plane) will have a $y$-component which is of the same sign as the upstream IMF $B_{y}$-component when the spacecraft location is situated to the 'left' $(y>0)$ in Figure 2. It is also expected that when the spacecraft is located far along the flank to the 'right' $(y<0)$, it will have a $B_{y}$-component which is opposite that of the upstream IMF. However, for some range of locations to the 'right', it is hypothesized that there will be a mixture of magnetosheath $B_{y}$ observations with either the same or opposite sign as the upstream IMF. Determining the range of locations where this 'mixing' occurs should provide better understanding of the divergence of the magnetic field as it drapes about the magnetopause when there exists a relatively strong IMF $B_{x}$-component.

The 23 Geotail magnetosheath intervals adjacent to the magnetopause / magnetosheath boundary layer are analyzed in context with the IMF direction. For each magnetosheath interval, the IMF, the Geotail location, and the magnetosheath field are all rotated about the $x$-axis such that the IMF lies in the $x y$-plane as depicted in Figure 2. The spacecraft locations are then scaled to be placed just outside the model magnetopause, as displayed in Figure 3. For the intervals for which the $y$-component of the spacecraft location is positive, 10 of 12 have the same sign of magnetosheath $B_{y}$ as is observed in the IMF (for the two intervals for which the sign is opposite, the IMF $B_{y}$ component is $<1 \mathrm{nT}$ ). This is as one might expect for draping of the IMF. For $-4<y_{\text {Geotail }}<0 R_{E}, 4$ of 5 intervals have the same sign of magnetosheath $B_{y}$ as is observed in the IMF. This is the same ratio (same sign / opposite sign) as for the $y_{G \text { Geotail }}>0$ intervals. For $y_{G e o t a i l}<-4 R_{E}$, there are an almost equal numberof intervals with the same sign (3 cases) as with the opposite sign (2 cases). This suggests that the divergence of the IMF at the 
magnetopause, even when the IMF is aligned rather closely with the solar wind flow direction, occurs rather far along the flank. This further implies that the schematic shown in Figure $2 b$ is the more likely draping configuration.

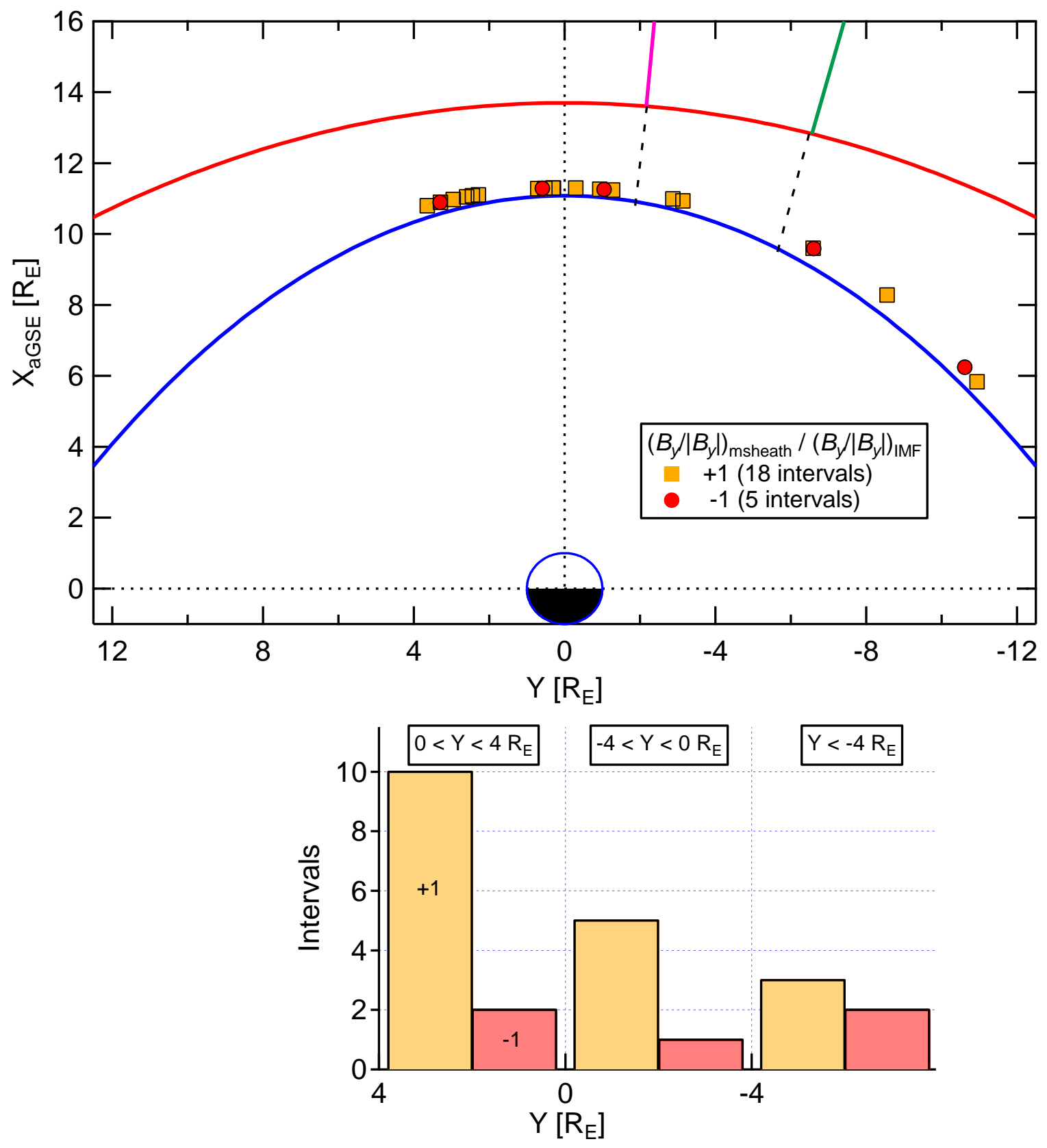

Fig.3. Top) Geotail magnetosheath intervals just upstream of the magnetopause. 18 intervals have the same sign of magnetosheath $B_{y}$ as the IMF; 5 intervals have the opposite sign. Bottom) Histogram of the intervals in the top panel, separated by ranges in $Y$. 


\section{Summary}

This brief study suggests that even when the IMF is relatively closely aligned with the solar wind flow (i.e., includes a relatively large $B_{x-a G S E}$ component), the divergence of the draped magnetic field occurs rather far downstream at the magnetopause. This result is expected to be of use for the development of analytic magnetosheath magnetic field models, as well as for global numerical models of the solar wind / magnetosphere interaction. However, a much more comprehensive study of this topic is needed. This could be accomplished with additional observations; e.g., from added low-latitude magnetopause crossings from the Geotail (late 2005 - present), ISEE-1,2, THEMIS, and MMS missions; and high-latitude crossings from the Cluster mission. Many of these observations would benefit from sampling of the unshocked solar wind closer to the bow shock (e.g., using THEMIS, MMS) to reduce the uncertainties in the propagation time. Although the occurrence of nearly flow-aligned IMF conditions are rare, such conditions are important for understanding how the solar wind interacts with the magnetosphere, and merits further investigation.

Appendix.

A derivation is provided of the location of intersection by the IMF normal to the bow shock surface. Using a simple conic section surface as described in Farris et al., [1991] (in 2D, for simplicity):

$r=\frac{r_{0}(1+\epsilon)}{1+\epsilon \cos \theta}$

where $\theta=\cos ^{-1}(x / r)$ (often referred to as the solar zenith angle).

Eq. 1 can be rewritten in terms of a scalar field $(u=u(x, y, z))$, such that the bow shock is then defined as a surface of constant value $(u=0)$. Explicitly, Eq.1 is rewritten as

$r_{0}(1+\epsilon)=r+\epsilon x=\sqrt{x^{2}+y^{2}}+\epsilon x$

or,

$r=\sqrt{x^{2}+y^{2}}=r_{0}(1+\epsilon)-\epsilon x$ 
Squaring both sides and moving all terms to one side, the bow shock surface is then described as:

$u=\left(1-\epsilon^{2}\right) x^{2}+2 \epsilon(1+\epsilon) r_{0} x-(1+\epsilon)^{2} r_{0}^{2}+y^{2}=0$

and the normal to the surface $(-\nabla u /|\nabla u|)$ can then be determined.

The IMF direction with respect to the $x_{\text {GGSE }}$ axis is then denoted by the angle $\psi$, and related to the normal of the bow shock surface:

$\hat{x} \cdot \hat{n}=\cos \psi=\frac{-\partial u / \partial x}{|\vec{\nabla} u|}$

Squaring both sides, using a trigonometric identity, and rearranging:

$\left(\frac{\partial u}{\partial y}\right)^{2}=\left(\frac{\partial u}{\partial x}\right)^{2} \tan ^{2} \psi$

or,

$y^{2}=(1+\epsilon)^{2}\left((1-\epsilon)^{2} x^{2}+2 \epsilon(1-\epsilon) r_{0} x+\epsilon^{2} r_{0}^{2}\right) \tan ^{2} \psi$

Replacing $y^{2}$ using Eq. A4:, and solving for $x$ (taking the positive root):

$a x^{2}+b x+c=0$,

where

$a=(1-\epsilon)\left(\left(1-\epsilon^{2}\right) \tan ^{2} \psi+1\right)$

$b=2 \epsilon\left(\left(1-\epsilon^{2}\right) \tan ^{2} \psi+1\right) r_{0}$

$c=(1+\epsilon)\left(\epsilon^{2} \tan ^{2} \psi-1\right) r_{0}^{2}$

and the distance orthogonal to the $x_{a G S E}$ axis is found from Eq A4.

Acknowledgments. The author wishes to thank the Geotail and Wind instrument and science teams for providing the observations to the public via CDAWeb. In addition, the author thanks Elle Allen, Katy Kuei, Marcos Pedreiro, and Janine Stovall for their efforts and contributions in identifying geophysical boundaries in the Geotail data sets. SMP was supported by NSF grant No. 1303186. 


\section{References}

Bargatze, L.F., R.L. McPherron, J. Minamora and D. Weimer (2005), A new interpretation of Weimer et al's solar wind propagation delay technique, J. Geophys. Res., 110, A07105, doi:10.1029/2004JA010902.

Farris, M., S.M. Petrinec, and C.T. Russell (1991), The thickness of the magnetosheath: Constraints on the polytropic index, Geophys. Res. Lett., 18, 1821-1824.

Farrugia, C.J., et al. (2010), Magnetosheath for almost-aligned solar wind magnetic field and flow vectors: Wind observations across the dawnside magnetosheath at $X=-12 R_{e}$, J. Geophys. Res., 115, A08227, doi:10.1029/2009JA015128.

Fuselier, S.A., K.J. Trattner, and S.M. Petrinec (2011), Antiparallel and component reconnection at the dayside magnetopause, J. Geophys. Res., 116, A10227, doi:10.1029/2011JA016888.

Greenstadt, E.W. and R.W. Fredricks (1979), Shock system in collisionless space plasmas, in Solar System Physics, III, edited by L.J. Lanzerotti, C. F. Kennel, and E. N. Parker, North-Holland, Amsterdam.

Haaland, S., G. Paschmann, and B.U.Ö. Sonnerup (2006), Comment on “A new interpretation of Weimer et al.'s solar wind propagation delay technique" by Bargatze et al., J. Geophys. Res., 111, A06102, doi:10.1029/2005JA011376.

Jelínek, K., Z. Němeček, J. Šafránková, J.-H. Shue, A. V. Suvorova, and D. G. Sibeck (2010), Thin magnetosheath as a consequence of the magnetopause deformation: THEMIS observations, J. Geophys. Res., 115, A10203, doi:10.1029/2010JA015345.

Kan, J.R., and D.W. Swift (1983), Structure of the quasi-parallel bow shock: Results of numerical simulations, J. Geophys. Res., 88(A9), 6919-6925, doi:10.1029/JA088iA09p06919.

Merka, J., A. Szabo, J. Safranková, and Z. Nemecek (2003), Earth's bow shock and magnetopause in the case of a field-aligned upstream flow: Observation and model comparison, J. Geophys. Res., 108, 12691278, doi:10.1029/2002JA009697. 
Neugebauer, M., and R. Goldstein (1997), Particle and field signatures of coronal mass ejections in the solar wind, in Coronal Mass Ejections, Geophys. Monogr. Ser., vol. 99, edited by N. Crooker, J. Joselyn, and J. Feynman, p. 245-251, AGU, Washington, D. C.

Neugebauer, M., R. Goldstein, and B. E. Goldstein (1997), Features observed in the trailing regions of interplanetary clouds from coronal mass ejections, J. Geophys. Res., 102, 19,743-19,751, doi:10.1029/97JA01651.

Pi, G., J.-H. Shue, J.-K. Chao, Z. Němeček, J. Šafránková, and C.-H. Lin (2014), A reexamination of longduration radial IMF events, J. Geophys. Res., 119, 7005-7011, doi:10.1002/2014JA019993.

Scholer, M., M. Fujimoto, and H. Kucharek (1993), Two-dimensional simulations of supercritical quasiparallel shocks: Upstream waves, downstream waves, and shock re-formation, J. Geophys. Res., 98, 18,971-18,984, doi:10.1029/93JA01647.

Shue, J.-H., J.-K. Chao, P. Song, J.P. McFadden, A. Suvorova, V. Angelopoulos, K.H. Glassmeier, and F. Plaschke (2009), Anomalous magnetosheath flows and distorted subsolar magnetopause for radial interplanetary magnetic fields, Geophys. Res. Lett., 36, L18112, doi:10.1029/2009GL039842.

Spreiter, J.R., and A.W. Rizzi (1974), Aligned magnetohydrodynamic solution for solar wind flow past the earth's magnetosphere, Acta Astron., 1, 15-35.

Suvorova, A. V., J.-H. Shue, A. V. Dmitriev, D. G. Sibeck, J. P. McFadden, H. Hasegawa, K. Ackerson, K. Jelinek, J. Šafránková, and Z. Němeček (2010), Magnetopause expansions for quasi-radial interplanetary magnetic field: THEMIS and Geotail observation, J. Geophys. Res., 115, A10216, doi:10.1029/2010JA015404.

Tang, B. B., C. Wang, and W. Y. Li (2013), The magnetosphere under the radial interplanetary magnetic field: A numerical study, J. Geophys. Res., 118, 7674-7682, doi:10.1002/2013JA019155. 
Trattner, K.J., J.S. Mulcock, S.M. Petrinec, and S.A. Fuselier (2007), Probing the boundary between antiparallel and component reconnection during southward interplanetary magnetic field conditions, $J$. Geophys. Res., 112, A08210, doi:10.1029/2007JA012270.

Trattner, K.J., S.M. Petrinec, S.A. Fuselier, and T.D. Phan (2012), The location of reconnection at the magnetopause: Testing the maximum magnetic shear model with THEMIS observations, J. Geophys. Res., 117, A01201, doi:10.1029/2011JA016959.

Trenchi, L., et al. (2008), Occurrence of reconnection jets at the dayside magnetopause: Double Star observations, J. Geophys. Res., 113, A07S10, doi:10.1029/2007JA012774.

Watari, S., M. Vandas, and T. Watanabe (2005), Solar cycle variation of long-duration radial interplanetary magnetic field events at 1 AU, J. Geophys. Res., 110, doi:10.1029/2005JA011165.

Weimer, D.R., and J.H. King (2008), Improved calculations of interplanetary magnetic field phase front angles and propagation time delays, J. Geophys. Res., 113, A01105, doi:10.1029/2007JA012452.

Weimer, D.R., D.M. Ober, N.C. Maynard, W.J. Burke, M.R. Collier, D.J. McComas, N.F. Ness and C.W. Smith (2002), Variable time delays in the propagation of the interplanetary magnetic field, J. Geophys. Res., 107, 10.1029/2001JA009102.

Weimer, D.R., D.M. Ober, N.C. Maynard, M.R. Collier, D.J. McComas, N.F. Ness, C.W. Smith and J. Watermann (2003), Predicting interplanetary magnetic field (IMF) propagation delay times using the minimum variance delay technique, J. Geophys. Res., 108, 1026, doi:10.1029/2002JA009405. 